\title{
Dose Dependences of the Conductivity and Luminescence in ZnSe Single Crystals
}

\author{
V.Ya. Degoda ${ }^{a, *}$, M. Alizadeh ${ }^{a}$, N.V. Martynyuk ${ }^{b}$ And N.Yu. Pavlova ${ }^{c}$ \\ ${ }^{a}$ Taras Shevchenko National University of Kyiv, Kyiv, Ukraine \\ ${ }^{b}$ Lviv Polytechnic National University, Lviv, Ukraine \\ ${ }^{c}$ National Pedagogical Dragomanov University, Kyiv, Ukraine
}

\begin{abstract}
The studies of time-resolved dose dependences of conductivity and luminescence in ZnSe crystals at various temperatures $(8,85,295$, and $430 \mathrm{~K})$ under X-ray and UV-excitation have revealed that dose dependences under X-ray excitation build up much more slowly and are more informative in comparison with UV-excitation. It is due to high penetrating depth of X-ray radiation and respective involvement of a large sample volume in the kinetic processes. Also, due to the local inhomogeneity of the excitation in the absorption of X-quantum, a significant share of the generated electron-hole pairs recombine in this local area creating a scintillation pulse, and not participating in the conductivity. The delay in the onset of the X-ray conductivity buildup at $8 \mathrm{~K}$ for several seconds is due to the high efficiency of the localization of free carriers in the traps, all of which become deep at this temperature. The different buildups of various bands of luminescence of irradiation time can be explained by not only different concentration of luminescence centers but also by their localization in various sections of free charge carriers. Dose dependences of the luminescence and conductivity also show that the scintillation pulse amplitudes and the current pulse amplitudes of the X-ray conductivity are not constant during irradiation of the ZnSe crystals.
\end{abstract}

DOI: $10.12693 /$ APhysPolA.133.984

PACS/topics: 72.20.-i, 78.55.Et, 29.40.Mc

\section{Introduction}

The wide-band-gap semiconducting ZnSe crystals [1,2] are excellent samples to study the physical processes that occur in the scintillation and semiconductor detectors in the absorption of the high-energy quanta. A detailed study of these processes is necessary for both improving the existing detectors, and creating new ones. Te-doped ZnSe crystals have proven to be good scintillators [3-6], and the high-resistivity ZnSe crystal with a resistivity $\rho \geq 10^{12} \Omega \mathrm{cm}$ can be used as the semiconducting detectors at room and high temperatures $[7,8]$. During the Xray and UV excitation, an intensive luminescence of two emission bands (with maxima at $630 \mathrm{~nm}$ and $970 \mathrm{~nm}$ ) is observed in ZnSe crystals [9-12], while a conductivity is easily registered [13-15].

Recently, the kinetic studies have become almost exotic and are mainly limited to the studies of the fast luminescence fading. Under kinetic studies in the general case, we mean changes in the luminescence intensity and the conduction current magnitude with the time of exposure and their change over time after the cessation of excitation. In our opinion, this is due to the low informative content of the kinetic measurements when only one research method is applied. High informative content of the kinetics occurs when a broad range of studies is using and so different methods complement each other [16]. The second essential point is to hold a comprehensive set

*corresponding author; e-mail: degoda@univ.kiev.ua of simultaneous studies. For example, to perform simultaneous recording of the luminescence and conductivity.

The changes of luminescence and conductivity in phosphorous crystals with irradiation time greatly depend not only on the concentration of various luminescence centers, but also on the concentration of different traps. Studying the time-resolved dependencies of luminescence and conductivity, one can use the fact that at different temperatures there are different ratios between the shallow and deep traps that should change the nature of the luminescence and conductivity curves. An important feature of these measurements is the simultaneous registration of the luminescence intensity of both emission bands and the magnitude of the conduction current.

The purpose of the study was to obtain the experimental dose dependences of luminescence and conductivity at different temperatures using X-ray and UV excitation and get explanations to the observed regularities. Under the dose dependence we mean the dependence of the conduction current and the emission intensity on constant exposure time starting from initial moment of the excitation $(t=0)$.

\section{Methodology of the experiment}

The conductivity and luminescence of ZnSe single crystals under X-ray quanta excitation and excitation by optical quanta with an energy greater than the band-gap (band-gap excitation) were investigated. To obtain crystals with a minimum impurity concentration and a maximum resistivity $\left(\rho \geq 10^{12} \Omega \mathrm{cm}\right.$ ), a specially unalloyed ZnSe crystals were grown from the purified batch. Let us note that the concentration of free electrons in such crystals is $10^{2}-10^{4} \mathrm{~cm}^{-3}$. The studies were performed using 
two high-resistance ZnSe crystals (sample \#1 and \#2) with various concentrations of the recombination centers.

Combined studies of luminescence and conductivity of ZnSe specimens under X-ray and UV-excitation were carried out. At excitation temperatures of 8, 85, 295, and $430 \mathrm{~K}$, we have investigated the buildup of the conductivity and luminescence of two dominant broad bands with maxima at 630 and $970 \mathrm{~nm}$, their emission spectra of photoluminescence (PL) and X-ray luminescence (XRL), dependences of thermally stimulated luminescence (TSL) and thermally stimulated conductivity (TSC). The sample was placed in a vacuum cryostat allowing utilization of various temperatures ranging from 8 to $500 \mathrm{~K}$. Different excitation temperatures utilization makes it possible to vary the concentration ratio between shallow and deep traps in the sample.

To study the conductivity, the single crystals were sprayed with two 3-layer metallic contacts by a resistive method. The chemical composition of each layer was specially selected to obtain ohmic contacts with excellent adhesion. The electrical contacts were rectangular strips of $5 \mathrm{~mm}$ length and $1 \mathrm{~mm}$ width, at a distance of $5 \mathrm{~mm}$ from each other. A stabilized voltage from 0 to $1000 \mathrm{~V}$ was applied to one electrical contact, while the other was grounded via a nanoammeter. The following condition was satisfied for all values of the conduction current: the input impedance of a nanoammeter was several orders of magnitude smaller than the electrical resistance of a ZnSe sample. We have selected a test voltage $U_{0}$ from the initial linear region of the voltage-ampere characteristics XRC and PC. Preliminary, using a thermo-emf method we found out that samples have a dark $n$-type conductivity.

An X-ray excitation was performed with integrated radiation of the X-ray tube BHV7 (Re, $20 \mathrm{kV}, 25 \mathrm{~mA}$ ) through the beryllium window of the cryostat in a perpendicular direction to the surface of the sample. The distance from the anode of the X-ray tube to the sample was $120 \mathrm{~mm}$, that provided a maximum X-ray intensity $I_{X}=0.635 \mathrm{~mW} / \mathrm{cm}^{2}$.

For the photoexcitation, we used 7 ultraviolet UF-301 light emitting diodes (maximum irradiation wavelength of $395 \mathrm{~nm}$ ) located on the same site $=20 \mathrm{~mm}$ and having a mutual power supply. The power supply unit $7 \times$ LED allowed varying of the stabilized current value up to $180 \mathrm{~mA}$. The emission of each LED was directed on the sample through the quartz window of the cryostat from a distance of $55 \mathrm{~mm}$. The intensity of the UV-excitation of the sample was $I_{P L}=0.156 \mathrm{~mW} / \mathrm{cm}^{2}$.

The conduction current and the luminescence radiation of the sample were recorded simultaneously. Simultaneous registration allows obtaining of more detailed and reliable information about the processes occurring in the sample. The luminescence was recorded via two channels (at $45^{\circ}$ angles to normal): integrally and spectrally. The integral luminescence of the sample that passed through one of the two quartz windows of the cryostat (if necessary through an optical filter) was focused with a quartz lens on the photocathode of PMT-106. Through another quartz window of the cryostat, the luminescence radiation went via a high-speed monochromator (MDR2) with the quartz condensers and was recorded with PMTs: PMT-106 in the visible region or PMT-83 in the IR region (in cooling mode). The integration constant of the PMT current amplifiers and the conduction current (nanoammeter) was $1 \mathrm{~s}$. All spectra were corrected taking into account the spectral sensitivity of the recording system. Upon terminating the excitation, the phosphorescence and current relaxation were registered for a specified time, then the sample was heated with a constant rate of $\approx 0.3 \mathrm{~K} / \mathrm{s}$ and TSL (two registration channels) and TSP were registered.

\section{The results of the experiment}

\subsection{The luminescence spectra of ZnSe single crystals}

The spectra of PL and XRL in these high-resistivity ZnSe samples practically do not differ in the spectral position of the maxima, in the half-widths and the ratio of bands intensities. Also, it should be noted that the total PL intensity at the excitation intensity of $0.156 \mathrm{~mW} / \mathrm{cm}^{2}$ and low temperatures $(8$ and $85 \mathrm{~K}$ ) differs little from the XRL integrated intensity at the excitation intensity of $0.635 \mathrm{~mW} / \mathrm{cm}^{2}$. Moreover, this overall luminescence intensity is only a few times weaker than the luminescence intensity of a classic luminophore $\mathrm{ZnS}-\mathrm{Cu}$, indicating the low concentrations of nonradiative recombination centers in the high-resistance ZnSe crystals. The characteristic luminescence spectra are shown in Fig. 1.

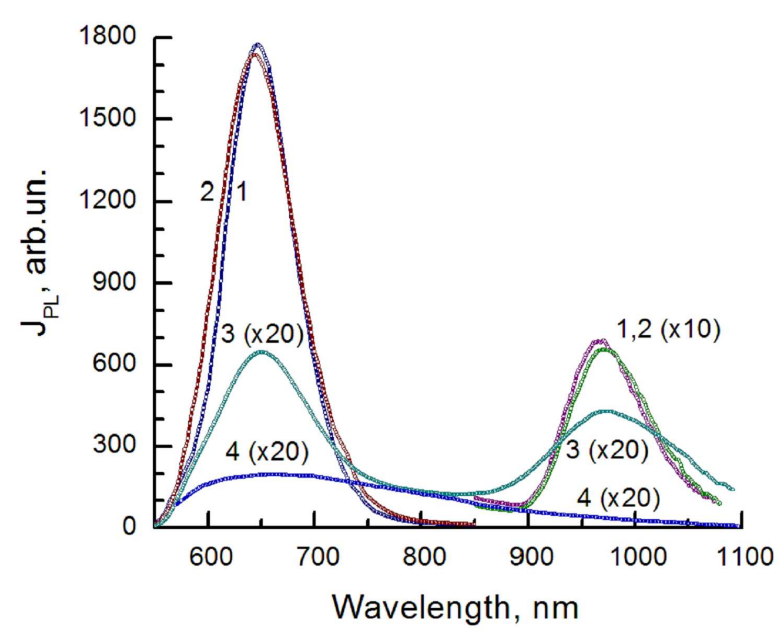

Fig. 1. PL spectra of the ZnSe sample $(\# 1)$ at temperatures: $8 \mathrm{~K}(1), 85 \mathrm{~K}(2), 295 \mathrm{~K}(3), 430 \mathrm{~K}(4)$ ). (Curves 1 and 2 in the IR region are multiplied by 10 , curves 3 and 4 are multiplied by 20.).

\subsection{Dose dependences of the ZnSe luminescence and conductivity}

Dose dependences of XRC and XRL obtained at $8 \mathrm{~K}$ (Fig. 2) show, firstly, a noticeable buildup over the time 
from the start of the X-ray irradiation and, secondly, significant difference in buildup for XRC current and various XRL bands. For XRC current, we even observed a lag in the buildup for almost $2 \mathrm{~s}$. Even after $20 \mathrm{~min}$ of X-ray irradiation, being a high irradiation dose $\left(0.76 \mathrm{~J} / \mathrm{cm}^{2}\right)$, a steady state equilibrium is not reached.

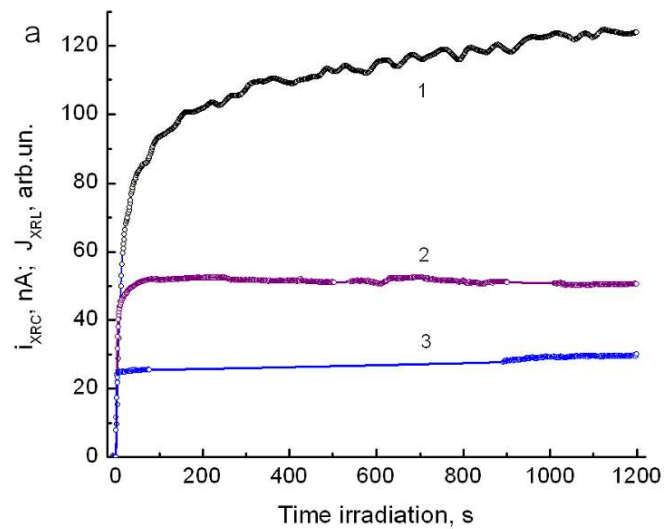

b

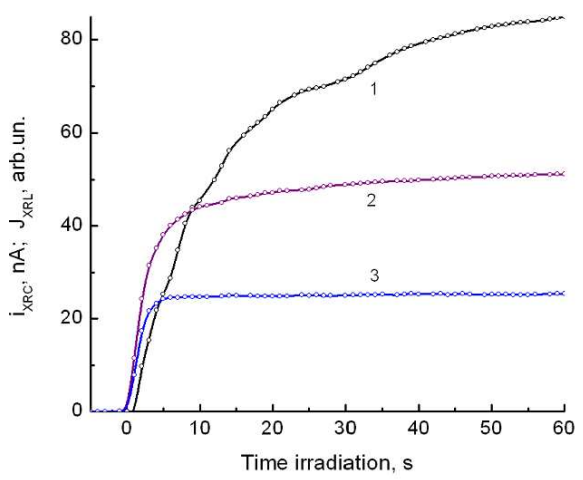

Fig. 2. Dose dependences (a) and their initial sections (b) of XRC (1) and XRL of the bands $630 \mathrm{~nm}(2)$ and $970 \mathrm{~nm}(3)$ of the ZnSe sample $(\# 1)$ at $8 \mathrm{~K}$ and $U_{0}=$ $4 \mathrm{~V}$.

Similar dose dependences of the PC and PL under UVirradiation basically duplicate a behavior of the excitation intensity $I_{P L}(t)$ (Fig. 3). The required buildup of the $\mathrm{PC}$ and $\mathrm{PL}$ corresponds to $1 \mathrm{~s}$ of amplifier integration.

Thus, the buildup of the conduction current and the luminescence intensity of irradiation time at a low temperature depends on a type of excitation.

To study the resulting lag in buildup of the XRC current, experimental measurements of the buildup of XRC and XRL of irradiation time at various intensities of the X-ray excitation (Fig. 4) were held at $85 \mathrm{~K}$ on ZnSe sample $(\# 2)$. It reveled a noticeable lag of the XRC current at a low-intensity $I_{X}$.

The situation changes drastically at room temperature (295 K), when both XRC current and XRL luminescence reach the maximum values within a few seconds (Fig. 5). Moreover, these few seconds are due to the time constant

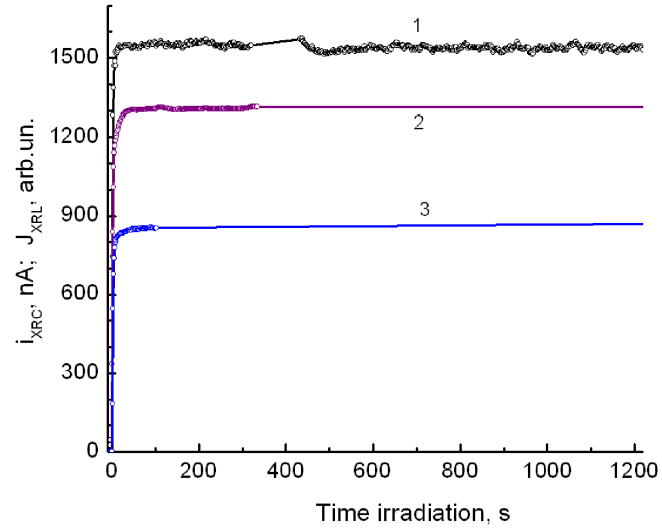

Fig. 3. Dose dependences of the PC (1) and PL of the bands $630 \mathrm{~nm}(2)$ and $970 \mathrm{~nm}$ (3) of the ZnSe sample $(\# 1)$ at $8 \mathrm{~K}$ and $U_{0}=4 \mathrm{~V}$.
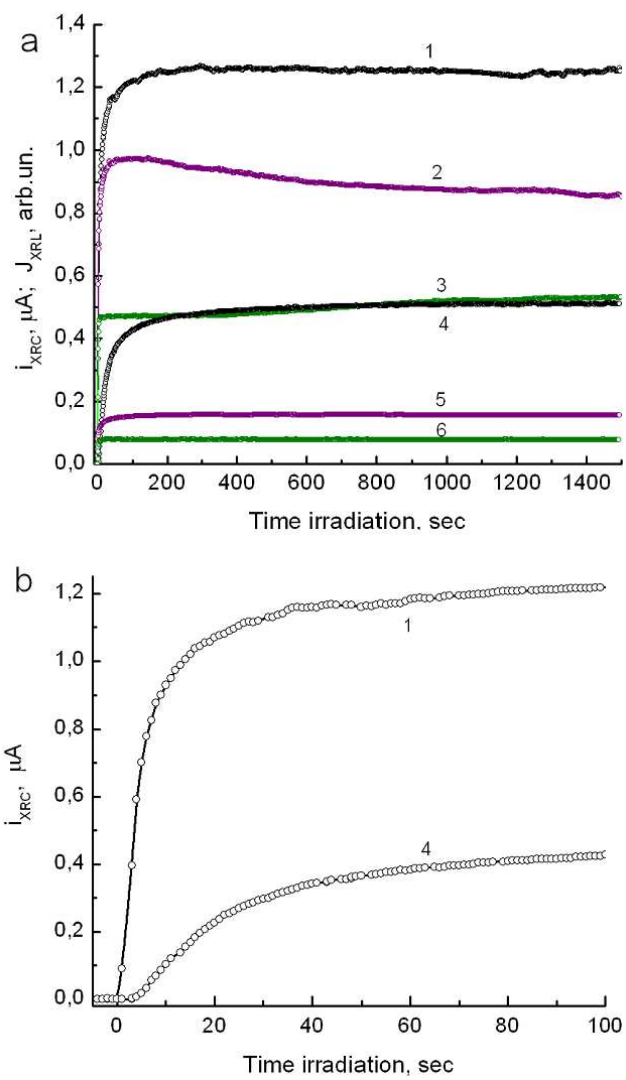

Fig. 4. Dose dependences of the XRC $(1,4)$, XRL of the bands $630 \mathrm{~nm}(2,5)$ and $970 \mathrm{~nm}(3,6)$ at $85 \mathrm{~K}$ and $U_{0}=12 \mathrm{~V}$ at different intensities of the X-ray excitation: $I_{X}=0.635 \mathrm{~mW} / \mathrm{cm}^{2}(1,2,3)$ and $I_{X}=0.127 \mathrm{~mW} / \mathrm{cm}^{2}$ $(4,5,6)$ of the ZnSe sample (\#2) (a), the initial sections of the XRC (b). 
of the registration amplifiers $\left(\tau_{r s}\right)$. However, the initial parts of the XRC and XRL curves are well approximated by the relation: $i_{X} / i_{0}=J_{X} / J_{0}=\left[1-\exp \left(-t / \tau_{r s}\right)\right]$.

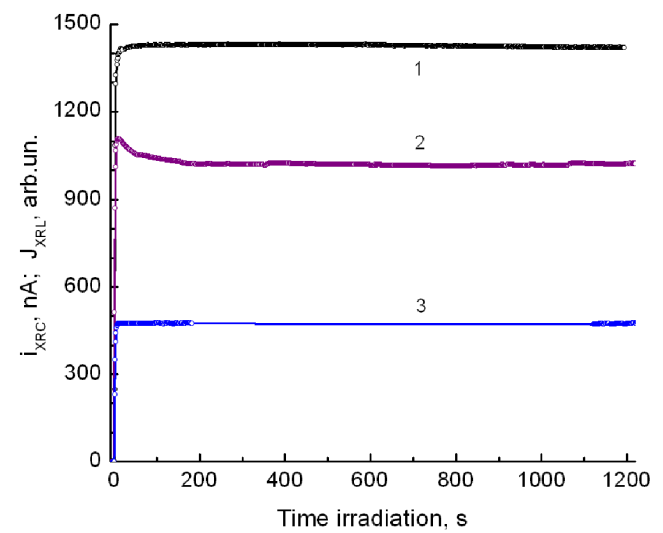

Fig. 5. Dose dependences of the XRC (1) and XRL of the bands $630 \mathrm{~nm}(2)$ and $970 \mathrm{~nm}(3)$ of the ZnSe sample $(\# 1)$ at $295 \mathrm{~K}$ and $U_{0}=40 \mathrm{~V}$.

A similar situation was observed for the UV excitation. Figure 6 shows the initial parts of the dose dependences $i_{P L}$ and $J_{P L}$ obtained at room temperature. It should be noted that due to thermal luminescence quenching, the XRL and PL intensities at $295 \mathrm{~K}$ are noticeably weaker than at $85 \mathrm{~K}$.

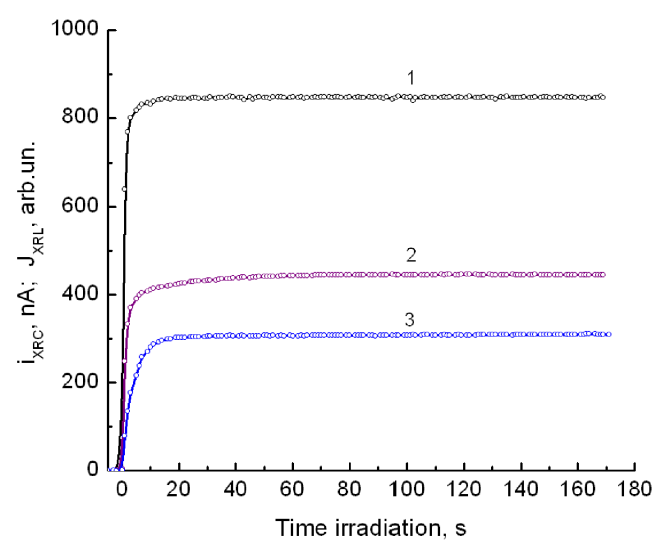

Fig. 6. Dose dependences of the PC (1) and PL of the bands $630 \mathrm{~nm}(2)$ and $970 \mathrm{~nm}$ (3) of the ZnSe sample $(\# 1)$ at $295 \mathrm{~K}$ and $U_{0}=12 \mathrm{~V}$.

It is quite obvious that the changes of luminescence and conductivity with irradiation time are determined by the concentrations of the recombination centers and the concentrations of the traps. The presence of the centers of the radiative recombination (luminescent centers) is determined by luminescence spectra, whereas the presence of traps is determined by the TSL and TSC curves. Therefore, it is necessary to conduct TSL and TSC studies to determine a temperature at which the deep traps are absent.
At $430 \mathrm{~K}$, the luminescence band $970 \mathrm{~nm}$, due to thermal quenching, is no longer recorded in the spectra. At this temperature, only the XRC current buildups with $\tau \approx 10 \mathrm{~s}$, while XRL $(630 \mathrm{~nm}), \mathrm{PL}(630 \mathrm{~nm})$ and PC reach maximum value almost instantly $\left(\tau_{r s}\right)$ (Figs. 7 and 8) and remain constant during the excitation.

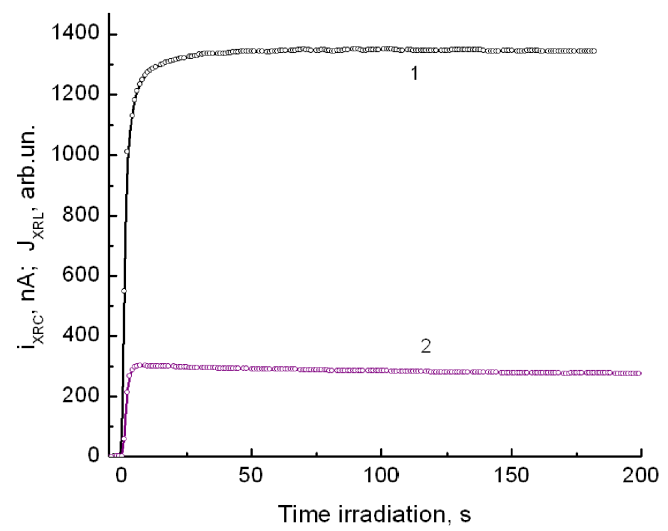

Fig. 7. Dose dependences of the XRC (1) and XRL of the $630 \mathrm{~nm}$ band $(2)$ of the ZnSe sample $(\# 1)$ at $430 \mathrm{~K}$ and $U_{0}=40 \mathrm{~V}$.

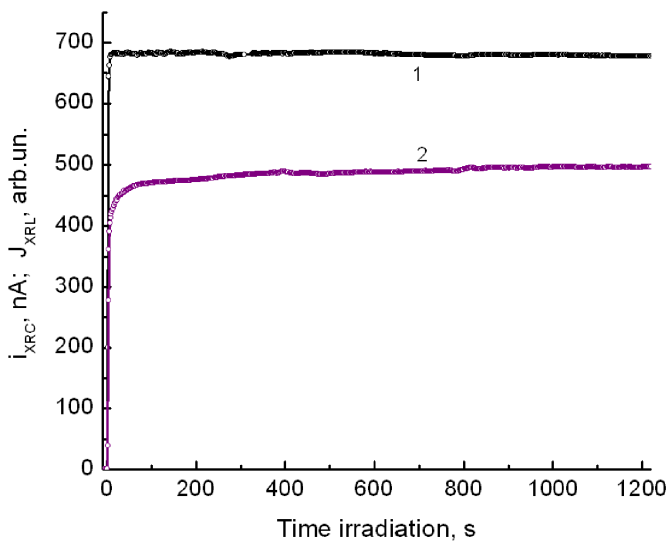

Fig. 8. Dose dependences of the PC (1) and PL of the bands $630 \mathrm{~nm}(2)$ and $970 \mathrm{~nm}$ (3) of the ZnSe sample $(\# 1)$ at $430 \mathrm{~K}$ and $U_{0}=12 \mathrm{~V}$.

Thus, the difference in behaviors of XRC, XRL, PC and PL dose dependences can be explained by the accumulation of free charge carriers in traps, i.e. accumulation of the light sum in the ZnSe crystals.

\section{3. $T S C$ and TSL in $Z n S e$}

The accumulation of charge carriers during crystals excitation occurs only in the phosphorescent and deep traps. It is known that in the low-temperature region (up to $300 \mathrm{~K}$ ), we observe TSL in ZnSe crystals [17, 18]. Above room temperature, there are deep traps in the ZnSe crystals, as evidenced by the curve 1 in Fig. 7 and 
small bends in the TSC curve. The buildup of the XRC and XRL dose dependences is caused by the accumulation of charge carriers on the local centers. It should be noted that concentrations of the recharged traps and recombination centers do not remain constant during and after the irradiation (Fig. 9). These concentrations increase monotonically during irradiation, and when after irradiation, decrease monotonically with the observed phosphorescence and relaxation of the conduction current. Moreover, the traps of different depth $\left(E_{i}\right)$ are emptied with different speeds (Fig. 9) as they have different thermal delocalization probability: $w_{i}=w_{i 0} \exp (-$ $\left.E_{i} / k T\right)$. The registered TSL and TSC curves with different holding times between the cessation of excitation and the beginning of heating (Fig. 9) confirm that the thermal delocalization of carriers from the traps occurs during the irradiation process. However, this additional increase in the concentration of free carriers during irradiation can be neglected.

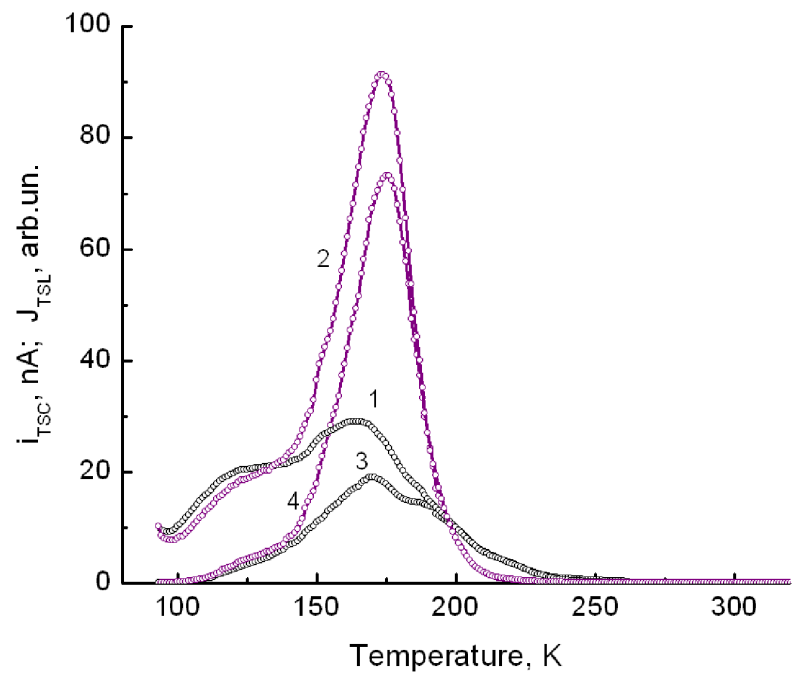

Fig. 9. TSC $(1,3)$ and TSL $(2,4)$ curves of the ZnSe sample $(\# 1)$ after a similar dose of the X-ray irradiation at $85 \mathrm{~K}$, but after different phosphorescence duration: $<1 \mathrm{~min}(1,2)$ and $1 \mathrm{~h}(3,4)$.

Thus, to obtain the correct dose dependences, the sample must be preheated in the dark to a temperature when all traps are thermally emptied (no accumulated light sum). The ZnSe crystals must be preheated to $450 \mathrm{~K}$.

\section{Discussion}

A noticeable difference in XRC and XRL buildup of irradiation time and their difference from the $\mathrm{PC}$ and $\mathrm{PL}$ at low temperatures in the ZnSe crystals can be explained by the different nature of the excitation. When one Xray quantum is absorbed, $h v_{X} /(2.5 \div 3) E_{g}$ thousands of "hot" free electron-hole $(\mathrm{e}-\mathrm{h})$ pairs are generated in the material [19]. A radius of the generation region $\left(r_{g}\right)$ is defined by the ratio [19]:

$$
r_{g}=\frac{6 \pi \varepsilon_{0}^{2} \sqrt{E_{g}\left(h v_{X}\right)^{3}}}{e^{4} n \ln \left(h v_{X} / 3 E_{g}\right)},
$$

(where $n$ is the total electron concentration in the semiconductor), that for many materials gives the radius of the generation region of the order of a few dozens of nm. Further, a rapid $\left(t<10^{-11} \mathrm{~s}\right)$ thermalization of the free carriers occur (free electrons and a hole lose excess of kinetic energy). Despite the difference in effective mass of electrons and holes up to 10 times, due to the Coulomb interaction between thousands of electrons and thousands of holes, the size of the regions of finding these electrons and holes are substantially identical and does not even reach one $\mu \mathrm{m}$. An absorption coefficient $\left(k_{X}\right)$ in $\mathrm{ZnSe}$ for $h v_{X}=13 \mathrm{keV}$ (the maximum of $\mathrm{X}$-ray Re-tube Bremsstrahlung radiation at a voltage of $20 \mathrm{kV})$ is $k_{X} \approx 250 \mathrm{~cm}^{-1}$. This means that the depth of the excitation region is $\approx 40 \mu \mathrm{m}$. As a result, under X-ray excitation $\left(I_{X}=0.635 \mathrm{~mW} / \mathrm{cm}^{2}\right)$, there are about $4.9 \times 10^{14} \mathrm{~cm}^{-2} \mathrm{~s}^{-1} \mathrm{e}-\mathrm{h}$ pairs generated in the depth of the sample under the unit surface area per unit time. There is a slightly different mechanism for the generation of free $\mathrm{e}-\mathrm{h}$ pairs under UV excitation. Upon the absorption of a single UV quantum with an energy greater than the width of the band-gap in the semiconductor, a transition of the electron from the depth of the valence band to the conduction band occurs. That is to say one free $\mathrm{e}-\mathrm{h}$ pair is generated, while the generated electron and a hole have the kinetic energy greater than thermal (thermally equilibrium) velocity. However, during time of less than $10^{-11} \mathrm{~s}$, the thermalization takes place due to scattering on point defects of the crystal structure and phonons of the lattice, and both a free electron and a hole lose excess of kinetic energy above the equilibrium energy. Absorption of UV quanta takes place in a thin surface layer due to huge absorption coefficient $\left(k_{P L}>10^{4} \mathrm{~cm}^{-1}\right)$. This area is slightly extended due to the diffusion of the thermal motion of free charge carriers, but remains $\approx 1 \mu \mathrm{m}$. It is easy to estimate the total number of the generated $\mathrm{e}-\mathrm{h}$ pairs in the surface area under the unit sample surface per unit time, when $I_{P L}=0.156 \mathrm{~mW} / \mathrm{cm}^{2}: I_{P L} / h v_{P L}=3 \times 10^{14} \mathrm{~cm}^{-2} \mathrm{~s}^{-1}$. One can see that under these excitation intensities, the total number of the generated free $\mathrm{e}-\mathrm{h}$ pairs is equal for both types of excitation. Since the lifetime of free electrons and holes does not depend on the nature of the excitation, the concentration of the free carriers will be 25 times greater under UV excitation. A number of sites on which the free charge carriers (traps) can be localized is $\approx 40$ times less under UV excitation. Since the PC and PL dose dependences at all temperatures buildup during less than $1 \mathrm{~s}$, it is obvious that these traps are being filled during time less than $1 \mathrm{~s}$, which is due to a large concentration of UV generated $\mathrm{e}-\mathrm{h}$ pairs. It particularly explains the experimental fact that the maximum value of the accumulated light sum under X-ray excitation is ten times more than under prolonged UV excitation (it was determined by the intensity of TSL curves). Natu- 
rally, we should not forget about the possibility of the highlight action of the exciting UV radiation. Thus, the dose dependences under X-ray excitation buildup much more slowly and are more informative.

An important difference of the X-ray excitation from the UV-excitation is a spatial microinhomogeneity of the electronic excitations generation [19] while absorbing Xray quantum. Thousands of generated $\mathrm{e}-\mathrm{h}$ pairs are in a small area with a diameter less than $0.5 \mu \mathrm{m}$. The Coulomb interaction between the electrons and holes prevents the rapid expansion of this field [20]. As a result, if a material contains a sufficient amount of recombination centers [21], as our ZnSe samples (Fig. 1), a large portion of the free charge carriers will recombine in this local area creating a scintillation pulse. It is confirmed experimentally by the dose dependences of the luminescence at low temperatures (Figs. 2 and 4). Under the X-ray irradiation, the luminescence intensity increases abruptly to a value of $0.65 \div 0.75$ of the maximum intensity value. It also means that not all of the generated free charge carriers take part in the conductivity. XRC current buildups slower than the XRL bands intensity and there is even a time lag (see Figs. $2 \mathrm{~b}$ and $4 \mathrm{~b}$ ) of about $2 \mathrm{~s}$. At $8 \mathrm{~K}$ in ZnSe, the traps of all types are deep and blank in the initial moment of X-irradiation. The initial intensive filling of traps is responsible for this XRC current lag.

Thus, the comparative analysis of the generation of the free $\mathrm{e}-\mathrm{h}$ pairs in ZnSe crystals under X-ray excitation and UV-excitation jointly with the analysis of physical processes of XRC, XRL, PC, and PL allows explaining the basic patterns of the experimental dose dependences of the conductivity and luminescence.

\section{Conclusions}

1. A comparison of the dose dependences of the conductivity and luminescence at X-ray and UV excitations shows that the X-ray excitation is more informative to investigate the influence of traps on the luminescence and conductivity.

2. Due to the micro heterogeneity of the X-ray excitation, not all generated free charge carriers are involved in conductivity. A significant amount of them (up to $65 \div 75 \%$ ) immediately recombine at the luminescence centers in the excitation micro-regions, creating a scintillation pulse.

3. The initial buildup lag of the conduction current, when compared to the luminescence buildup, may be attributed to the intense filling of the traps by the free charge carriers in the first seconds of irradiation at low temperatures.

4. Differences in luminescence intensity buildup for various bands are due to their different concentrations and various capture cross-sections of the free charge carriers.

5. The studies of the dose dependences of the luminescence and conductivity at X-ray excitation show that the amplitude of the scintillation pulse and the amplitude of the current pulse change during irradiation. This fact puts considerably higher demands to the purity of crystals if using them as scintillating and semiconducting analyzers of the ionizing radiation spectrum.

\section{References}

[1] G. Watkins, in: Point Defects of Lattice, Mir, Moscow 1979.

[2] I. Broser, V. Kutzer, B. Lummer, R. Heitz, Ch. Fricke, A. Hoffmann, J. Menninger, H. Hartman, E. Krause, J. Cryst. Growth 138, 75 (1994).

[3] V. Ryzhikov, N. Starzhinskiy, K. Katrunov, L. Gal'chinetskii, Funct. Mater. 9, 135 (2002).

[4] I. Dafinei, M. Fasoli, F. Ferroni, E. Mihokova, F. Orio, S. Pirro, A. Vedda, IEEE Trans. Nucl. Sci. 57, 1470 (2010).

[5] V.D. Ryzhikov, N.G. Starzhinskiy, L.P. Gal'chinetskii, L.L. Nagomaya, P.A. Gashiu, V.P. Makhniy, G. Tamulaitis, W. Klamra, D.N. Kozin, E.A. Danshin, IEEE Trans. Nucl. Sci. 48, 356 (2001).

[6] Yun Ho Cho, Se Hwan Park, Woo Gyo Lee, Jang Ho Ha, Han Soo Kim, N. Starzinskiy, Dong Hoon Lee, Sangsoo Park, Yong Kyun Kim, J. Nucl. Sci. Technol. 45, 534 (2008).

[7] A.O. Sofiienko, V.Y. Degoda, Radiat. Measur. 47, 27 (2012).

[8] M.S. Brodin, V.Ya. Degoda, B.V. Kozhushko, A.O. Sofiienko, V.T. Vesna, Radiat. Measur. 65, 36 (2014).

[9] V.D. Ryzhikov, N.G. Starzhinskiy, L.P. Gal'chinetskii, V.I. Silin, G. Tamulaitis, E.K. Lisetskaya, Int. J. Inorg. Mater. 8, 1227 (2001).

[10] F.J. Bryant, P.S. Manning, J. Phys. C 5, 1914 (1972).

[11] N.K. Morozova, I.A. Karetnikov, V.V. Blinov, E.M. Gavrishchuk, Semiconductors 35, 512 (2001).

[12] N.K. Morozova, I.A. Karetnikov, V.V. Blinov, E.M. Gavrishchuk, Semiconductors 35, 24 (2001).

[13] V.Y. Degoda, A.O. Sofiienko, Semiconductors 44, 1 (2010).

[14] V.Ya. Degoda, G.P. Podust, Semiconductors 50, 579 (2016).

[15] V.Y. Degoda, N.Yu. Pavlova, G.P. Podust, A.O. Sofiienko, Physica B Condens. Matter 465, 1 (2015).

[16] V.Ya. Degoda, A.F. Gumenjuk, Yu.A. Marazuev, Kinetics of Luminescence and Conductivity on Phosphor Crystals, Publishing House "Kyiv University", Kyiv 2016.

[17] B.G. Markey, S.W.S. McKeever, Phys. Status Solidi A 138, 225 (1993).

[18] V. Degoda, A. Gumenjuk, N. Pavlova, A. Sofiienko, S. Sulima, Acta Phys. Pol. A 129, 304 (2016).

[19] V.Y. Degoda, A.O. Sofiienko, Ukr. J. Phys. 52, 255 (2007).

[20] A.O. Sofiienko, V.Ya. Degoda, V.N. Kilin, Global J. Frontier Res. Phys. Space Sci. 12, 9 (2012).

[21] V.Ya. Degoda, V.G. Pivovarenko, I.M. Moroz, D.Yu. Shilov, J. Lumin. 165, 174 (2015). 